\title{
ON THE REINHARDT-MAHLER THEOREM
}

\section{RAJINDER JEET HANS}

1. Let $A_{1}, \cdots, A_{n}$ be $n$ linearly independent points in $R_{n}$, the $n$-dimensional Euclidean space. The set $\Lambda=\left\{u_{1} A_{1}+\cdots+u_{n} A_{n}\right.$ : $u_{1}, \cdots, u_{n}$ integers $\}$ is called a lattice, and $\left\{A_{1}, \cdots, A_{n}\right\}$ is called a base of $\Lambda$. Let $A_{i}$ have co-ordinates $a_{1 i}, \cdots, a_{n i}$. Then $d(\Lambda)$ $=\left|\operatorname{det}\left(a_{i j}\right)\right|$ is called the determinant of the lattice $\Lambda$; it is independent of the choice of a base of $\Lambda$.

Let $S$ be a set in $R_{n}$. A lattice $\Lambda$ is said to be $S$-admissible if $\Lambda$ has no point other than the origin 0 in the interior of $S$. The critical determinant $\Delta(S)$ of $S$ is defined by $\Delta(S)=\inf d(\Lambda)$, where $\Lambda$ runs over all $S$-admissible lattices $(\Delta(S)=\infty$ if $S$ has no admissible lattice). Clearly, $S \subset T$ implies $\Delta(S) \leqq \Delta(T)$.

One of the principal problems in Geometry of Numbers is to find a method for determining $\Delta(S)$ for a given set $S$. For two- and threedimensional symmetrical ${ }^{1}$ convex bodies, Minkowski reduced the problem to the discussion of special classes of lattices with points on the boundary of the body. This method has been partially extended to symmetrical convex bodies in $R_{4}$, but it is very difficult to apply in spaces of dimension higher than two. Reinhardt [7] and Mahler [4] independently proved that for a symmetrical convex domain $K$ in $R_{2}$, $\Delta(K)=H(K) / 4$, where $H(K)$ denotes the area of a smallest symmetrical "hexagon" ${ }^{2}$ containing $K$. In other words, a symmetrical convex domain can be inscribed in a space-filling symmetrical convex domain with the same critical determinant. The straightforward generalization of this result to higher dimensions would be:

If $K$ is a symmetrical convex body in $R_{n}$ then $K$ is contained in a space-filling symmetrical convex body $\beta$ with $\Delta(K)=\Delta(P)=V(P) / 2^{n}$, where $V(\mathcal{P})$ denotes the volume of $P$.

The object of this note is to prove that this generalization does not hold in $R_{n}$, for $n \geqq 3$.

2. Let $\nVdash$ be the class of symmetrical open convex bodies in $R_{n}$.

Definition. Let $K \in \mathcal{K} . K$ is said to be $\mathcal{K}$-maximal (for packings) if $H \in \mathfrak{K}, H \nsupseteq K$ implies that $\Delta(H)>\Delta(K)$.

Definition. A $K$-admissible lattice $\Lambda$ with $d(\Lambda)=\Delta(K)$ is called a critical lattice of $K$.

Received by the editors July $28,1966$.

${ }^{1}$ By a symmetrical body we mean a body centered at 0 , unless the contrary is evident in the context.

${ }^{2}$ By a "hexagon" we mean a polygon with at most six sides. 
It is well known that if $K \in \mathcal{K}$, then $K$ has at least one critical lattice. Let $\Lambda$ be a critical lattice of $K$. One can easily construct (as in Davenport and van der Corput $[1$, pp. 410]) a polyhedron $\odot$ containing $K$, such that $\Lambda$ is $\rho$-admissible, and so $\Delta(\rho)=\Delta(K)(d(\Lambda)=\Delta(K)$ $\leqq \Delta(\rho) \leqq d(\Lambda))$. This implies that $\mathfrak{K}$-maximal bodies must be polyhedra. Now we prove

THEOREM 1. Let $\odot$ be a polyhedron. Then $\beta$ is $\mathcal{K}$-maximal iff every critical lattice of $\odot$ has at least one point in the $(n-1)$-dimensional interior of each $(n-1)$-dimensional face of $P$.

Proof. Suppose $\odot$ is $\mathcal{K}$-maximal and $\odot$ has a critical lattice $\Lambda$ with no point in the interior of an $(n-1)$-dimensional face $F$. Since $\Lambda$ is a discrete set, moving the faces $\pm F$ parallel to themselves we can find a larger set $\boldsymbol{\rho}^{\prime} \in \mathcal{K}$ for which $\Lambda$ is admissible. Since $\Delta\left(\mathcal{P}^{\prime}\right)=\Delta(\rho)$, this gives a contradiction to the definition of maximality.

Next suppose that every critical lattice of $\beta$ has a point in the interior of each $(n-1)$-dimensional face of $P$. Let $S \in \mathcal{K}, S \nsupseteq \odot$. Let $P \in S, P \notin \odot$. Join $O P$. Let $O P$ meet the boundary of $\rho$ at $P_{1}$. Then $P_{1} \in S$. Since $S$ is open, there is a neighborhood of $P_{1}$ contained in $S$. Consequently, we can find a point $P_{2}$ in $S$ which also lies in the $(n-1)$-dimensional interior of an $(n-1)$-dimensional face $F$ of $\rho$ containing $P_{1}$. Since $S$ contains the convex cover of $\rho$ and $P, S$ also contains the interior of the face $F$. Since $F$ contains in its interior a point of every critical lattice of $\beta$, no critical lattice of $\odot$ can be $S$ admissible. Hence, no lattice $\Lambda$ with $d(\Lambda)=\Delta(\odot)$ is $S$-admissible. Since $S$ possesses critical lattices, it follows that $\Delta(S)>\Delta(\odot)$. This proves that $\mathcal{P}$ is $\mathcal{K}$-maximal.

3. In this section we give an example of a polyhedron in $R_{3}$ which is $\mathcal{K}$-maximal but which is not space-filling.

Let $\beta$ be the octahedron

$$
|x|+|y|+|z|<1 \text {. }
$$

Then $V(P)=$ volume of $P=4 / 3$, while Minkowski [5] proved that

$$
\Delta(\odot)=\frac{19}{108} \neq \frac{1}{8} V(\odot) .
$$

Hence $\odot$ is not space-filling.

Minkowski [5] also proved that the only critical lattice of $\odot$ (up to automorphisms of $K$ ) is the lattice $\Lambda$ generated by the points $(-1 / 3,1 / 2,1 / 6),(1 / 6,-1 / 3,1 / 2)$ and $(1 / 2,1 / 6,-1 / 3)$. It is obvious that $\Lambda$ and hence all other critical lattices of $\rho$ have a point in 
the interior of each 2-dimensional face of $\boldsymbol{\rho}$. Therefore, by Theorem 1, $P$ is $\nVdash$-maximal.

Remark. This example was suggested by Professor C. A. Rogers. Our original example was the cut cube

$$
|x+y+z|<1 / 2, \quad|x|<1, \quad|y|<1,|z|<1
$$

whose critical lattices were determined by Whitworth [8].

4. We next prove

THEOREM 2. For each $n>2$, there exist $K$-maximal polyhedra in $R_{n}$ which are not space-filling.

As an immediate consequence we have

THEOREM 3. The straightforward generalization of the ReinhardtMahler theorem (stated in the introduction) to $R_{n}(n \geqq 3)$ is not true.

We need two lemmas.

Lemma 1. Let $K \in \nVdash$ be space-filling. Then every $(n-1)$-dimensional face of $K$ is a finite union of nonoverlapping $(n-1)$-dimensional symmetric convex bodies.

For $n=3$, this lemma was proved by Minkowski (see Hancock [2]). The same method applies in higher dimensions.

Let $V_{n}(K)$ denote the $n$-dimensional volume of a set $K$.

Lemma 2. A simplex can not be a finite union of nonoverlapping symmetrical convex bodies.

Proof (suggested by A. Heppes). Let $S$ be a simplex in $R_{n}$. Let $S$ be the convex cover of $(n+1)$ points $A_{0}, A_{1}, \cdots, A_{n}$. Since symmetrical convex bodies transform in to symmetrical convex bodies under linear transformations ( $Y=A X+B, A$ nonsingular), we can suppose that $A_{0}=O, A_{1}=(1,0, \cdots, 0), \cdots, A_{n}=(0, \cdots, 1)$.

Suppose $S$ is the union of a finite number of symmetrical convex bodies $K_{1}, \cdots, K_{m}$. Let $F$ be the face of $S$ contained in $x_{n}=0$, then

$$
0<V_{n-1}(F)=V_{n-1}\left(F \cap\left(U \bar{K}_{i}\right)\right)=\sum V_{n-1}\left(F \cap \bar{K}_{i}\right)
$$

and $V_{n-1}\left(F \cap \bar{K}_{i}\right)>0$ for at least one $i$, say $i=1$. So $K_{1}$ has a face $F_{4}$, of volume $V_{n-1}\left(F_{1}\right)=V_{n-1}\left(F \cap \bar{K}_{1}\right)>0$, contained in $x_{n}=0$. The parallel face $F_{2}$ lies in $x_{n}=h_{1}$, where $h_{1}>0$, and $V_{n-1}\left(F_{2}\right)=V_{n-1}\left(F_{1}\right)>0$. Since $K_{1} \subset S, h_{1}<1$. Hence there are $K_{i}$ which lie above $x_{n}=h_{1}$ (in the obvious sense) and whose closures $\bar{K}_{i}$ have points in common with the face $F_{1}$. Let these be $K_{2}, \cdots, K_{m_{1}}$. Then 


$$
\begin{aligned}
0<V_{n-1}\left(F_{2}\right) & =V_{n-1}\left(F_{2} \cap\left(\begin{array}{c}
m_{1} \\
2
\end{array} \bar{K}_{i}\right)\right) \\
& =\sum_{i=2}^{m_{1}} V_{n-1}\left(F_{2} \cap \bar{K}_{i}\right),
\end{aligned}
$$

so that there exists a set, say $K_{2}$, such that $V_{n-1}\left(F_{2} \cap \bar{K}_{2}\right)>0$. Therefore $K_{2}$ has a face $F_{3}$ in $x_{n}=h_{1}$ with $V_{n-1}\left(F_{3}\right)>0$. The parallel face $F_{1}$ of $K_{2}$ with $V_{n-1}\left(F_{4}\right)=V_{n-1}\left(F_{3}\right)>0$, is in $x_{n}=h_{2}$, where $0<h_{1}<h_{2}<1$ (since this face is contained in $S$ ). Repeating this argument, we would get a sequence $K_{1}, K_{2}, \cdots$ of sets $K_{i}$, and faces $F_{2}, F_{4}, \cdots$ lying in the planes $x_{n}=h_{1}, h_{2}, \cdots$, where $0<h_{1}<h_{2}<\cdots<1$. Since the $K_{i}$ 's are finitely many, this is impossible.

Proof of Theorem 2 . Let $K$ be the cube $\left|x_{i}\right|<1,1 \leqq i \leqq n$.Ollernshaw [6] proved that $K$ is irreducible in $\mathscr{K}$ i.e. if $H \in \mathscr{K}$ and $H \Phi K$ then $\Delta(H)<\Delta(K)$.

For $\epsilon>0$, consider

$$
K_{e}:\left|x_{i}\right|<1, \quad 1 \leqq i \leqq n, \quad\left|x_{1}+\cdots+x_{n}\right|<n-\epsilon .
$$

Then $\Delta\left(K_{e}\right)<\Delta(K)$. Fix an $\epsilon<2 /(n+1)$. Define

$$
\delta=\inf \left\{\epsilon^{\prime}: \epsilon^{\prime} \leqq \epsilon, \Delta\left(K_{\epsilon^{\prime}}\right)=\Delta\left(K_{\epsilon}\right)\right\} .
$$

By a theorem of Mahler [3], it follows that

$$
\Delta\left(K_{\delta}\right)=\Delta\left(K_{\epsilon}\right)<\Delta(K)=1 .
$$

Define

$$
K_{\delta, 1}:\left\{\begin{array}{l}
\left|x_{i}\right|<1, \quad 2 \leqq i \leqq n \\
\left|x_{1}+\cdots+x_{n}\right|<n-\delta
\end{array}\right.
$$

Then

$$
V\left(K_{\delta, 1}\right)=2^{n}(n-\delta)>2^{n} .
$$

Hence

$$
\Delta\left(K_{\delta, 1}\right)>1>\Delta\left(K_{\delta}\right)
$$

Therefore, by the same argument as above, there exists $k_{1} \geqq 1$ such that the critical determinant of $K_{\delta, 1} \cap\left\{\left|x_{1}\right|<k_{1}\right\}$ is equal to $\Delta\left(K_{\delta}\right)$ and $k_{1}$ is maximal with this property. Applying this argument successively to other coordinates we get a body

$$
H_{8}:\left|x_{i}\right|<k_{i}, \quad 1 \leqq i \leqq n, \quad\left|x_{1}+\cdots+x_{n}\right|<n-\delta,
$$


where $k_{i} \geqq 1, \Delta\left(H_{\delta}\right)=\Delta\left(K_{\delta}\right)$, and each $k_{i}$ is maximal with this property, i.e. if we expand $H_{\delta}$ by moving out any two parallel faces, the critical determinant is increased.

As in the proof of Theorem 1, one can show that every critical lattice of $H_{\delta}$ has a point in the interior of each $(n-1)$-dimensional face of $H_{\delta}$. Hence $H_{\delta}$ is $\mathcal{K}$-maximal. Now we shall prove that $H_{\delta}$ is not space-filling. By Lemmas 1 and 2, it is enough to prove that one face of $H_{\delta}$ is a simplex.

It can be easily shown that

$$
V\left(H_{\delta}\right) \geqq 2^{n}-2 \frac{\delta^{n}}{n}+2 \frac{k_{i-1}}{n}\left(2^{n-1}-\frac{\delta^{n-1}}{n-1}\right),
$$

for each $i$.

Since $V\left(H_{\delta}\right) \leqq 2^{n} \Delta\left(H_{\delta}\right)<2^{n}$, we must have $k_{i}<1+\delta, 1 \leqq i \leqq n$.

The $n$ points $P_{i}=\left(y_{1}^{(i)}, \cdots, y_{n}^{(i)}\right)$,

where

$$
\begin{aligned}
& y_{j}{ }^{(i)}=k_{j} \quad \text { if } j \neq i, \\
& y_{i}{ }^{(i)}=(n-\delta)-\sum_{j \neq i ; 1 \leq j \leq n} k_{j}
\end{aligned}
$$

are vertices of the plane face $x_{1}+\cdots+x_{n}=n-\delta$. We assert that these are the only vertices. Any other possible vertex would be $Q=\left(z_{1}, \cdots, z_{n}\right)$, where for some fixed $i$,

$$
\begin{aligned}
& z_{i}=(n-\delta)-\sum_{j \neq i} \delta_{j} k_{j}, \quad z_{j}=\delta_{j} k_{j}(j \neq i), \\
& \delta_{j}= \pm 1 \text { and at least one } \delta_{j} \text { is }-1
\end{aligned}
$$

Since

$$
\begin{aligned}
z_{i} & =(n-\delta)-\sum_{j \neq i} \delta_{j} k_{j} \\
& =(n-\delta)-\sum_{j \neq i} k_{j}+2 \sum^{\prime} k_{j}
\end{aligned}
$$

(where $\sum^{\prime}$ is taken over those $j$ for which $\delta_{j}=-1$ )

$$
\begin{aligned}
& \geqq(n-\delta)-(n-1)(1+\delta)+2 \sum^{\prime} k_{j} \\
& \geqq 3-n \delta>1+\delta \quad \text { (because } \delta<2 /(n+1)) \\
& >k_{i}
\end{aligned}
$$

it follows that $Q$ cannot be a vertex of $H_{\delta}$, and our assertion is proved. The points $P_{1}-P_{n}, P_{2}-P_{n}, \cdots, P_{n-1}-P_{n}$ have coordinates 


$$
\begin{aligned}
& \left(n-\delta-\sum k_{i}, 0, \cdots, 0, \sum k_{i}-(n-\delta)\right) \\
& \left(0,(n-\delta)-\sum k_{i}, 0, \cdots, 0, \sum k_{i}-(n-\delta)\right), \cdots \\
& \left(0, \cdots, 0, n-\delta-\sum k_{i}, \sum k_{i}-(n-\delta)\right)
\end{aligned}
$$

and are easily seen to be linearly independent.

Therefore, the face of $H_{\delta}$ in $x_{1}+\cdots+x_{n}=n-\delta$ is a simplex, and our proof is completed.

I want to express my gratitude to Professor R. P. Bambah for his kind help in preparing this note. I am also indebted to Professor A. Heppes, Professor C. A. Rogers, and Professor A. C. Woods for some useful suggestions.

\section{REFERENCES}

1. J. G. van der Corput and H. Davenport, On Minkowski's fundamental theorem in the geometry of numbers, Indag. Math. 8 (1946), 409-415.

2. H. Hancock, Development of Minkowski geometry of numbers, Macmillan, New York, 1939.

3. K. Mahler, On lattice points in n-dimensional star bodies (I): Existence theorems, Proc. Roy. Soc. London A (187) (1946), 151-187.

4. - On the minimum determinant and the circumscribed hexagon of a convex domain, Indag. Math. 9 (1947), 326-337.

5. H. Minkowski, Gesammelte Abhandlungen. II, Leipzig and Berlin.

6. K. Ollernshaw, Irreducible convex bodies, Oxford Quart. J. Math. (2) 4 (1953), 293-302.

7. K. Reinhardt, Über die dichteste gitterförmige Lagerung kongruenter Bereiche in der Eben usw., Abh. Math. Sem. Univ. Hansische 10 (1934), 213-231.

8. J. V. Whitworth, On the densest packing of sections of a cube, Ann. Mat. Pura Appl. (9) 27 (1948), 29-37. 